\title{
NUPR1 and its potential role in cancer and pathological conditions (Review)
}

\author{
TRACEY A. MARTIN, AMBER XINYU LI, ANDREW J. SANDERS, LIN YE, \\ KATHRYN FREWER, RACHEL HARGEST and WEN G. JIANG
}

Cardiff China Medical Research Collaborative, Cardiff University School of Medicine, Cardiff CF14 4XN, UK

DOI: 10.3892/ijo_xxxxxxxx

\begin{abstract}
Nuclear protein-1 (NUPR1) is also known as Com-1 or $\mathrm{p} 8$. It is a protein primarily found in the nucleus of various cells, including cancer cells, and it has been found to play an important role in cell stress and stress-related apoptosis. Over the past two decades, NUPR1 has been firmly indicated to play a role in the development and progression of numerous types of cancer, as well as in a number of other pathological conditions, including pancreatitis, diabetes, neurological and inflammatory conditions. The past decade has witnessed a rapid understanding of the biological and cellular mechanisms through which NUPR1 operates on cells and the identification of new variant of the protein. Most importantly, there have been comprehensive studies on the clinical and pathological aspects of NUPR1 and its variant in multiple malignancies and identification of therapeutic methods by targeting the protein. The present review aimed to summarise the current knowledge relating to NUPR1 in human malignancies and to discuss the associated controversies and potential future prospects of this molecule.
\end{abstract}

\section{Contents}

1. Introduction

2. Cellular function of NUPR1

3. NUPR1 in malignant disease

4. NUPR1 in other conditions

5. Therapeutic considerations

6. Challenges and future perspectives

Correspondence to: Dr Tracey A. Martin, Cardiff China Medical Research Collaborative, Cardiff University School of Medicine, Henry Wellcome Building, Heath Park, Cardiff CF14 4XN, UK E-mail: martinta1@cardiff.ac.uk

Key words: NUPR1, Com-1, p8, NUPR1-like, cancer, NUPR2, metastasis, survival, therapeutics

\section{Introduction}

Nuclear protein-1 (NUPR1; also known as Com-1 or p8) was initially discovered in 1997 and expressed as a small protein in the rat and as a novel gene that is activated in the acute phase of induced pancreatitis and during pancreatic development (1). The molecule, then named $\mathrm{p} 8$ was shown to be related to cell death and gene transcription. During the following 2 years, the same study group discovered the human version of $\mathrm{p} 8$, which shares a $74 \%$ similarity with rat $\mathrm{p} 8(1,2)$. Independently, in the same year, a second group discovered a new molecule in their search for candidate gene(s) associated with brain metastasis of breast cancer (3). In metastatic brain tumours, the expression of one gene was found to be elevated, compared with the parent breast cancer, and was named candidate of metastasis-1 or Com-1. It was soon found that these molecules were identical. In 2003, Quirk et al isolated a gene clone from pituitary derived cells and found it to encode $\mathrm{p} 8$ (4). These findings have since triggered active research into the role of this protein in cancer and other pathological conditions, such as pancreatitis. NUPR-2 or NUPR1-like protein was then identified (5), which appears antagonistic to some degree, to the function of NUPR1. Together, NUPRs indicate a fascinating area of research. The present review aimed to summarise the progress in studies on NUPR1, primarily in cancer and to a limited degree, in other pathological conditions.

\section{Cellular function of NUPR1}

There are some excellent reviews available in the literature which provide detailed knowledge of the molecular and cellular function of NUPR1 at the beginning of this decade $(6,7)$. The present review describes the advancements made in the understanding of NUPR1 in various cancer types, and some benign disorders, which have taken place since, and summarizes the key functions of NUPR 1 in cells, as well as associated genetic interactions, which are illustrated in Fig. 1.

Transcriptional regulations of NUPR1. NUPR1 is a transcription regulator protein with its gene located on chromosome 16 . It is typically expressed in response to stress signals induced by genotoxic signals and agents. Transforming growth factor $\beta$ (TGF $\beta$ ) is an important regulator of NUPR1 transcription. TGF $\beta$, upon binding to its receptor, initiates a canonical 
cascade, in which phosphorylated small mothers against decapentaplegic (SMAD)-2/3 proteins form a heteromeric complex with cofactor SMAD-4 and translocates into the nucleus. By binding on to the promotor at the 5'-untranslated region (5'-UTR), it elevates the transcription of NUPR1 through binding, a regulation appearing at a rapid pace (8).

DNA damage and repair. NUPR1 influences cancer cell resistance to metabolic stress-induced glucose starvation and hypoxia through the downstream regulation of Aurora kinase A (AURKA) expression. The inhibition of AURKA triggers a cytotoxin that can lead to DNA damage (9). NUPR1 is also important in $\gamma$-irradiation-induced damage and repair (10). It negatively controls DNA repair following $\gamma$-irradiation in the presence of MSL complex subunit 1 (MSL1) by regulating histone acetyltransferase (HAT) activity and is involved in intercommunication with p53 binding protein (P53BP1) (10). It has also been shown that the inhibition of NUPR1 by the organic synthetic molecule, ZZW-115, sensitizes cells to DNA damage, resulting in the reduction of SUMOylation of several proteins involved in DNA damage response by inhibiting the nuclear translocation of NUPR1. This decreases the SUMOylation-dependent functions of proteins involved in the DNA damage response (6).

Cell stress and cell death. NUPR1 regulates cellular damage and death in different forms, depending on the cell context and the types of stress induced. NUPR1 is involved in D9-tetrahydrocannabinol (THC)-induced cancer cell death through the downstream targeting of death inducible telomere repeat-binding factor 3 (TRB3) protein, transcription factor activating transcription factor 4 (ATF-4) and the protein $\mathrm{C} / \mathrm{EBP}$ homologous protein (CHOP) following endoplasmic reticulum stress (ERS) elevated from the synthesis of ceramide and collectively provokes apoptosis (11). NUPR1 downregulation in hepatocellular carcinoma (HCC) cells can enhance cell sensitivity to sorafenib treatment, which further controls cell growth through the RELB/IER3 pathway (12). The previous study by Santofimia-Castaño et al (2018) demonstrated that cell death observed following the knockdown of NUPR1 expression could be reversed by incubation with necrostatin-1, but not by the inhibition of caspase activity (13). The authors of that study thus described a model in which inactivation of NUPR1 in pancreatic cancer cells resulted in ERS that induced a mitochondrial malfunction, a deficient ATP production and, as consequence, cell death mediated by a programmed necrosis (13).

Cell growth, autophagy and death. NUPR1 promotes the proliferation of cancer cells by influencing cell cycle progression. NUPR1 can aid cells to enter the $\mathrm{S}$ phase by bypassing the G0/G1 checkpoint. Escape from the G0/G1 phase is achieved by an association between NUPR1 and cyclin inhibitory proteins resulting in downregulation of $\mathrm{p} 21$ and p57 (14). The knockdown of NUPR1 is able to regulate autophagy by interfering with FoxO3, promoting Bnip3 transcription in the control of autophagy, a stress-dependent self-defence mechanism that helps cells eliminate the toxic microenvironment (15). It has been reported that NUPR1 silencing suppresses autophagic activities and induces autophagy-mediated apoptosis in multiple myeloma (MM) cells through the PI3K/AKT/mammalian target of rapamycin (mTOR) pathway, which exhibits potential as a treatment strategy for MM (16), and that NUPR1 is a potent regulator of autolysosomal dynamics and is required for the progression of certain epithelial cancers as it regulates the late stages of autolysosome processing through the induction of the synaptosomal-associated protein (SNAP)-receptor (SNARE) protein synaptosomal-associated protein, $25 \mathrm{kDa}$ (SNAP25), which forms a complex with the lysosomal SNARE-associated protein, VAMP8. NUPR1 depletion deregulates autophagic flux and impairs autolysosomal clearance, inducing massive cytoplasmic vacuolization and premature senescence in vitro and tumour suppression in vivo (17).

Cell senescence. It has been found that in disordered pancreatic mouse cells, the inhibition of NUPR1 facilitates Kras-induced cellular senescence through the genomic downregulation of Dnmt1 expression, an enzyme transferring methyl groups onto DNA, which in turn decreases DNA methylation, crucial for transformation that helps the induction of Kras-dependent pancreatic cancer (18). NUPR1 can directly regulate the expression of DNA (cytosine-5)-methyltransferase 1 (Dnmt1) by interfering with its transcription process by binding on to the promoter (19). The silencing of NUPR1 promotes stress-induced senescence upon the enlarging flattened phenotype of cells provoking spatial pressure (14). As discussed above, NUPR1 is aberrantly expressed in a subset of cancer cells and predicts low overall survival rates for patients with lung cancer. NUPR1 depletion deregulates autophagic flux and impairs autolysosomal clearance, inducing massive cytoplasmic vacuolization and premature senescence (17).

Endothelial cells. As previously discussed by Cai et al (2016) in the context of methamphetamine (METH)-induced endothelial apoptosis, NURP1 functions as a fundamental regulator throughout the whole process (20). METH-associated disruptive effects give rise to the formation of ERS for compensating cell damage in endothelial cells. NUPR1 elevates transcription factor CHOP production in response to ERS, which then couples to the PUMA promoter through the mediation of p53, a downstream protein after the nupr1/chop axis. The succeeding cascades are achieved based on the presentation of NUPR1. The action of CHOP dissociates anti-apoptotic BCL-2 and upregulates pro-apoptotic BAX, as well as altering the membrane potential of the mitochondria. The resulting increased $\mathrm{BAX} / \mathrm{Bcl}-2$ ratio drives the apoptogenic factor cytochrome $c$ importing from mitochondria into the cell cytosol, which then successfully triggers endothelial caspase-mediated cell death. A previous study by Tang et al (2015) also revealed the importance of a reciprocal association between NUPR1 and ER stress by investing in Shigella enterotoxin (Shiga) toxin-induced enterocyte apoptosis (21).

Influence on metabolism. In 2018, Santofimia-Castaño et al examined NUPR1 depletion cooperating with ERS, inducing pancreatic cancer cell apoptosis and programmed necrosis by mediating cellular metabolism. Following NUPR1 knockdown, there was a decrease in ATP production, resulting in deficient oxygen availability (13). Mitochondrial membrane 


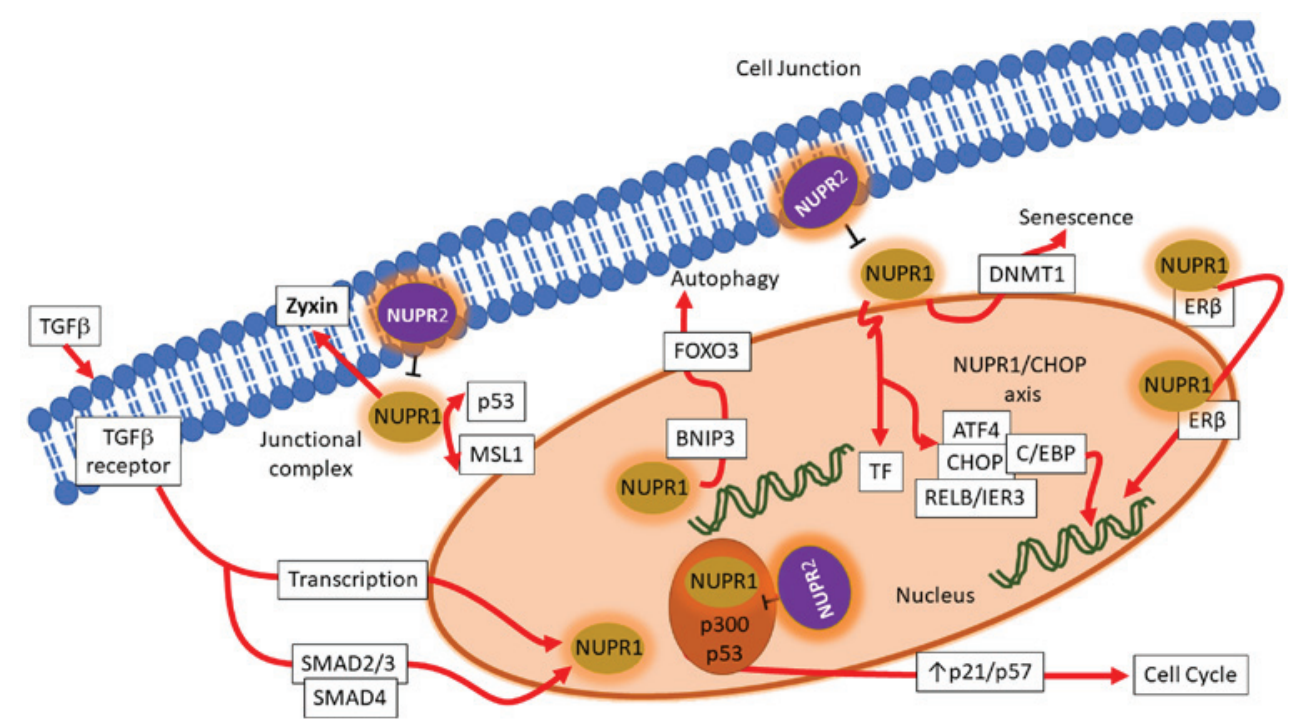

Figure 1. Schematic summarizing the key functions and interactions of NUPR1 in cells. NUPR1, nuclear protein-1. NUPR2 has been shown to block the activity of NUPR1 where indicated in the diagram.

potential disruption following $\mathrm{Ca}^{2+}$ uptake from the cytoplasm is also initiated by a shortage of NUPR1 together with ERS. The updated mitochondria content triggers the alteration in membrane permeability resulting in the discharge of cytochrome $c$, which leads to cell death (20). Other research has elucidated a confirmed association between the deficiency of NUPR1 and bone metabolism, by mediating the receptor activator of nuclear factor kappa-B ligand or (RANK ligand or RANKL) and sclerostin, which in turn enhances the proliferation of osteoblasts and the downregulation of osteoclasts (22). NUPR1 interacts with and activates SNAP25 to initiate an autolysosomal process that results in premature senescence and autophagy process (17). NUPR1 is an essential regulator in protein metabolism and glucose homeostasis (23). It interacts with p300 and Pax 2 through which it regulates the transactivation activity of Pax $2 \mathrm{~A}$ and Pax2B and influences the promoter activities of the glucagon gene (24). It is also a mediator of glucose induced growth of beta cells in the pancreas (25). It is also downstream of Zyxin, an adhesion junctional regulator protein with a controversial role in cancer (26).

\section{NUPR1 in malignant disease}

Overall involvement in cancer. Investigations into the role of NUPR1 have increased since the discovery that it was linked to brain metastasis in breast cancer (3). By injecting the human breast cancer cell line, MA-11, into athymic rats, brain metastases were established. In comparisons between the primary cancer cells and metastatic breast cancers of the brain using differential RNA display and protein analysis, NUPR1 was found to be present in metastatic cells and not in primary cancer cells and was found to be in aggressive MDA MB-231, but not in MCF-7 cells which are less aggressive (3). The authors of that study also demonstrated that there was a rapid rise in establishing a clinical link between NUPR1 and the development, progression and clinical outcome in various types of cancer. The tumorigenic effect of NUPR1 was subsequently demonstrated in that embryonic fibroblasts from $\mathrm{NUPR}^{+/+}$mice, when transformed with ras V12 mutation became tumorigenic and spread into the peritoneal cavity of mice compared with the same transformation of NUPR $1^{-1-}$ fibroblasts, which were non-tumorigenic $(27,28)$. It was surprising to note that $\mathrm{NUPR}^{+/+}$cells with ras V12 mutation transformation grew at a slower rate than the $\mathrm{NUPR}^{-/}$cells with ras V12 mutation transformation, in clear contrast to the in vivo results in the same study (28). Another observation made by the same authors was that the NUPR1-deficient cells grew more rapidly than the NUPR $1^{+/+}$cells (27). The clear discrepancy and disconnection between in vitro cell growth and in vivo tumour growth remain unexplained.

However, these early observations have driven a marked interest in examining the role of the molecule in individual human cancers. A summary of some of the key findings from previous studies is presented in Table I.

Breast cancer. In breast cancer cells, NUPR 1 has been shown to form a complex with $\mathrm{p} 300$ and $\mathrm{p} 53$ together with the p21 promoter, to upregulate the expression of $\mathrm{p} 21$, allowing breast cancer cell to progress through the cell cycle (29). In MCF-7 breast cancer cells, a HSP9 chaperone protein $\mathrm{p} 23$ was shown to result in 7.6 fold downregulation, although the response to oestradiol was less prominent (30). It has been reported that breast cancer cells have less nuclear staining than normal cells, but more cytoplasmic staining in mammary tumour tissues (31). A study on gene transcripts also demonstrated that NUPR1 was reduced in aggressive tumours and that high levels of NUPR 1 transcript were associated with a longer survival (31). The finding that NUPR1 levels in oestrogen receptor (ER) and ER $\beta$-negative tumours has an important bearing in survival led us to a further discovery that NUPR1 plays an interactive role with ER $\beta$ and that $17-\beta$-oestradiol is able to impact the cellular location of NUPR1 in breast cancer cells (32). Using genetic analysis to detect copy numbers of NUPR1 and ERBB2 (her2), Jung et al (2012) found that a gain of copy numbers of both genes in early breast cancer 
Table I. NUPR1 in clinical cancers.

\begin{tabular}{llll}
\hline Cancer type $\quad$ Methods applied & Clinical relevance
\end{tabular}

Breast cancer $\quad$ Northern blot analysis $(n=81)$

IHC and transcript analysis $(n=120)$

Genetic analysis of early stage breast cancer $(n=145)$

Gene transcript by PCR $(\mathrm{n}=96)$

Pancreatic cancer

Colorectal cancer

Cholangio-carcinoma IHC $(n=10)$

Prostate cancer IHC

Bladder cancer

Lung cancer

Endometrial cancer

Multiple myeloma (MM)
IHC and quantitative gene transcript analysis $(n=80)$

NUPR 1 transcript analysis by PCR $(n=50)$

IHC and PCR (38 pancreatic cancer, 5 liver metastasis and 7 metastatic lymph nodes) IHC $(n=44)$

IHC on pancreatic ductal adenocarcinoma ( $n=34$, TMA)

$\mathrm{IHC}(\mathrm{n}=36)$

IHC $(n=37)$

Quantitative gene transcript analysis

IHC ( $n=118)$, NSCLC (non small cell lung cancer)

IHC ( $n=198)$ and gene transcript analysis $(n=32)$

Geodata analysis $(n=152)$

Bone marrow from MM (N=4) and normal
High levels in tumours. No correlations with clinical and pathological parameters nor with uPA (urokinase-type plasminogen activator) and UPAR (urokinase-type plasminogen activator receptor)

Reduced nucleus and increased cytoplasmic staining in cancer cells. High level is linked to good prognosis

Simultaneous gain of NUPR1 and ERBB2 (receptor tyrosine-protein kinase erbB2 precursor) gene copy number indicate poorer clinical outcome

Stepwise increase of NUPR1 mRNA from normal, stage 1,2, 3 and 4 .

Tumour tissues stained positive for $\mathrm{p} 8$ and normal tissue mostly negative.

Highly positive in nodal positive tumours and is inversely correlated with the presence of apoptotic cells. No correlation with survival. Level of the NUPR1 expression, together with hypoxia inducible factor 1 subunit $\alpha$ (HIF1 $\alpha$ ) are inversely correlated with survival time NUPR1 is linked with cannibalism of pancreatic cancer which in turn linked to prognosis

Tumour tissues had higher levels of NUPR1 transcript than normal tissues. High stage tumours had less NUPR1. NUPR1 protein was more visible in nucleus in normal epithelial and in tumour cells stronger staining in the cytoplasm NUPR1 mRNA was highly raised in tumour tissues than normal tissues

NUPR1 mostly nuclear staining in ductal epithelial cells, increased nuclear staining in cholangiocarcinoma cells

Reduced nucleus and cytoplasmic staining in prostate cancer cells, compared with normal prostate epithelial cells

Mainly cytoplasmic staining, with invasive cancer cells stained less intensively

High levels of NUPR1 mRNA in adenocarcinoma, squamous cell carcinoma and adenosquamous carcinoma compared with the adjacent normal tissues

High level staining of NUPR1 in cancer tissues and high staining associated with shorter survival. High levels of NUPR1 protein and mRNA in deep tumours compared with superficial tumours

High levels of NUPR1 mRNA in MM than in normal 
Table I. Continued.

\begin{tabular}{|c|c|c|c|}
\hline Cancer type & Methods applied & Clinical relevance & (Refs.) \\
\hline \multirow[t]{4}{*}{$\begin{array}{l}\text { Hepatocellular } \\
\text { carcinoma (HCC) }\end{array}$} & Gene transcript analysis $(\mathrm{N}=23)$ & $\begin{array}{l}\text { A portion of the tumours ( } 4 \text { out of } 23 \text { ) has high } \\
\text { level }\end{array}$ & $(62)$ \\
\hline & $\begin{array}{l}\text { IHC and gene array }(\mathrm{n}=35 \text { including } \\
\text { normal liver, non-tumour and tumour } \\
\text { tissues) }\end{array}$ & $\begin{array}{l}\text { Increase in NUPR1 staining in liver cancer } \\
\text { (strong in nucleus and also with cytoplasmic } \\
\text { staining). Transcription ratio (tumour to normal) } \\
1.667(\mathrm{P}<0.005)\end{array}$ & $(63)$ \\
\hline & $\begin{array}{l}\text { HCC }(n=21) \text {, cirrhotic liver }(n=3) \\
\text { and normal liver }(n=3) \text { by IHC and } \\
\text { qPCR. }\end{array}$ & $\begin{array}{l}\text { HCC with high levels of nuclear staining of } \\
\text { NUPR1 and NUPR } 1 \text { mRNA than normal liver } \\
\text { tissues }\end{array}$ & $(12)$ \\
\hline & NUPR1 transcript analysis $(\mathrm{n}=158)$ & $\begin{array}{l}\text { Significantly higher in } \mathrm{HCC} \text { than normal liver } \\
\text { tissues and in tumours with high vascular } \\
\text { invasion. Combined expression of NUPR } 1 \text { and } \\
\text { thyroxin receptors significantly correlated with } \\
\text { the survival of the patients }\end{array}$ & $(66)$ \\
\hline \multirow[t]{2}{*}{ Thyroid cancer } & $\mathrm{IHC}(\mathrm{n}=150)$ & $\begin{array}{l}\text { Most normal and tumour tissues positive for } \\
\text { staining and tumour tissues tended to be } \\
\text { over-expressed. Anaplastic type less intense in } \\
\text { staining than papillary and follicular types. Large } \\
\text { tumours and those with lymph node } \\
\text { involvement more intense. }\end{array}$ & $(47)$ \\
\hline & IHC $(n=30)$ medullary carcinoma & $\begin{array}{l}43.4 \% \text { regarded as highly expressed and high } \\
\text { degree of staining linked to lymph node } \\
\text { metastasis and recurrence }\end{array}$ & $(48)$ \\
\hline Glioma & IHC and QPCR $(n=122)$ & $\begin{array}{l}\text { High levels of NUPR } 1 \text { mRNA seen in glioma } \\
\text { tissues than normal tissues. High levels staining } \\
\text { is associated with shorter survival of the patients. }\end{array}$ & $(68)$ \\
\hline Osteosarcoma & QPCR $(n=58)$ & $\begin{array}{l}\text { Osteosarcomas have significantly high levels of } \\
\text { NUPR1 transcript than non-tumour tissues. }\end{array}$ & $(67)$ \\
\hline
\end{tabular}

IHC, immunohistochemistry; qPCR, quantitative polymerase chain reaction; mRNA, messenger ribonucleic acid; PCR, polymerase chain reaction.

was a valuable predictor for patients with early-stage breast cancer (33). In animal models, NUPR1 together with BMP4, Cyr6, plod2 and angiopietin2, were shown to be markedly downregulated in transcription factor E2F knockdown mice and that collectively, these reductions were thought to contribute to the retardation of metastasis and presence of circulating cancer cells (34).

A more recent study has revealed that NUPR1 was essential for tumour repopulating cells in breast cancer cells (MCF-7) to grow, forming colonies and tumours in vivo (35). That study demonstrated that NUPR1 overexpression suppressed nestin and human telomerase reverse transcriptase (hTERT), both clonogenic markers, via the p53 pathway, leading to the inhibition of repopulation by this small population of cancer cells and a reduction in tumour growth in vivo, together revealing a tumour suppressor role for NUPR1 in breast cancer and indeed ovarian cancer (35). NUPR1 has been found to be present at high levels in breast cancer cells metastasised to bone, although not those to the brain, when compared with the parent cells (36). This is an interesting finding and that, together with a recent study demonstrating that NUPR1 may be linked to the osteoclastic activities of bone (22), may suggest that NUPR1 plays a pivotal role in bone metastasis from breast cancer. This possibility is strengthened by findings that NUPR1 is also an important factor in the growth of bone marrow mesenchymal cells (37).

Colorectal cancer. Colorectal tumour tissues exhibit high levels of the NUPR1 transcript $(38,39)$ and more cytoplasmic NUPR1 staining, compared with normal tissues (38). High stage tumours exhibited a less obvious presence of NUPR1. The same team demonstrated that the knockdown of NUPR1 from colorectal cancer cells ( $\mathrm{RKO}$ and $\mathrm{CaCo} 2)$ resulted in less growth, less colony formation and increased apoptosis, with minimal roles played in cellular migration, presenting a somewhat contrasting role to the clinical findings $(39,40)$; Wang et al demonstrated that the knockdown of NUPR1 reduced invasiveness and that the overexpression of NUPR1 exerted opposite effects (39). It appears the NUPR1-like 
protein (NUPR2 and NUPR11) plays a contrasting role to NUPR1 in colorectal cancer cells since the suppression of NUPR11 by miR2277 results in an increase in cell migration and cell growth (41).

Pancreatic cancer. Early reports of the clinical significance of NUPR1 in pancreatic cancer came from Su et al $(42,43)$, which demonstrated that pancreatic cancers, particularly metastatic and node-positive tumours exhibited high levels of NUPR1 protein, although no association with survival was established. A very compelling investigation revealed that the staining of NUPR1 protein in pancreatic ductal adenocarcinoma was inversely associated with the clinical outcome of patients over a 24-month follow-up period (44). That study also revealed that NUPR1, together with RelB and IER3, formed a group of markers not only for predicting patient outcome, but also in the development of intraductal neoplasia of the pancreas (44). Furthermore, NUPR1, together with hypoxia-inducible factor $\alpha(\mathrm{HIF} \alpha)$ and AURKA participated in the regulation of pancreatic cancer cell autophagy response to hypoxia and glucose deprivation (9). The loss/deletion of NUPR1 would result in the malfunction of the mitochondria due to ERS, leading to cell death (13). The pancreatic cancer cell line, colo357, is amongst the most sensitive cell types to a marked increase in NUPR 1 expression in the response to TGF $\beta 1$ (8). In pancreatic cancer, NUPR1 is intimately involved in homotypic cannibalism, or cell-in-cell, in that there is virtually no NUPR1. The cell-in-cell phenomenon can be enhanced by TGF $\beta$ in PANC1 cells (45). Using an elegant Pdx1-cre; LSL-Kras ${ }^{\text {G12D }}$; Ink4a/Arf ${ }^{\text {f/fl }}$ (KIC) mouse model, Cano et al (2014) demonstrated that a proficient NUPR 1 expression resulted in the development of murine pancreatic ductal adenocarcinoma. However, the deletion of the NUPR1 gene in these mice, although causing substantial perinatal death due to NUPR1 and Ink4a/Arf inactivation, the surviving mice exhibited a prolonged survival and less pancreatic cancer-related deaths (46). Pancreatic cancer cells derived from NUPR1-proficient KIC mice displayed a high degree of stemness and anchorage-independent growth compared with NUPR1-deficient cells (46).

Thyroid cancer. Normal thyroid tissue tends to have a low degree of NUPR1 staining while thyroid tumours have high levels (47). Papillary and follicular tumours stain stronger than anaplastic tumours and normal tissues. Node positive tumours also stain stronger than node-negative tumours (47). One of the most interesting observations from the study is the cellular protein location. In normal follicles, NUPR1 is exclusively displayed as nuclear staining. The same nuclear staining is observed in follicular tumours. However, papillary tumours largely stain the cytoplasmic region, particularly in those large tumour and tumours with lymph node metastasis (47). The majority of medullary tumours stain strongly for NUPR1 and high levels are linked to lymph node metastasis and recurrence (48).

Urological cancers. In a limited study on prostate cancer, nuclear and cytoplasmic staining were found in normal prostate epithelial cells and both staining patterns were reduced in prostate cancer cells (49). The knockdown of NUPR1 in
PC3, DU145 and CAHPV10 prostate cancer cells resulted in an increase in the invasiveness of the cells in their response to an invasion inducer, hepatocyte growth factor (HGF) (49). Bladder transitional cells largely have cytoplasmic staining, with invasive cancer staining at a lower intensity (50). The knockdown of NUPR1 from bladder cancer cell lines (RT112 and EJ138) results in an increase in both cell growth and invasiveness. In multiple bladder cancer cell lines, CUPR1 is one of the few epigenetically upregulated non-CpG island genes, together with TIMP1, TNFRSF14, ITGB4 and downregulated genes including MMP11 and FGF18 (51).

Lung cancer. Non-small cell lung cancer (NSCLC) tissues exhibit markedly high levels of NUPR 1 protein staining compared with adjacent normal tissues and those with high levels in tumours have a significantly shorter overall survival (28 months), a marked difference from those with low levels (80 months) (17). There is otherwise no significant association with tumour staging, smoking or age. Tumour tissues (adenocarcinoma, squamous cell carcinoma and mixed type) have significantly higher levels of NUPR1 transcript than their normal counterpart tissues (52). The same group have also shown that multiple human lung cancer cell lines, namely A549, SKME1, 95-D, NCI-H460, H1299, all highly express NUPR1 (52). In these lung cancer cells, knock-down of NUPR1 by siRNA results in the cells forming less colonies, becoming more apoptotic and forming less tumours in in vivo models $(17,52)$. It is also a key bystander response gene in the lung cancer cell line, H1299, when irradiated (53) and a responsive gene that is downregulated following the knockdown of a mitochondrial protein c3orf1 (translocase of inner mitochondrial membrane domain-containing protein 1) from the lung cancer cell line, 95D (54). In this case, the reduction in NUPR1 expression was also linked to a change in the cell cycle and the reduction in cellular migration.

Skin cancers. In K14 $\Delta$ NLef1/K14L61Rac1 double-transgenic mice, an exclusive skin tumour type, sebaceous carcinoma-like tumours are prevalent and are characterised by aggressive growth and progression (55). A previous study identified NUPR1 as one of the few responsive genes contributing to tumour progression as the result of RAC1 knockdown. In melanoma, the BRAF inhibitor, encorafenib, increased the expression of ATF4, CHOP and NUPR1 and induced the expression of PUMA (56).

Gynaecological cancers. In endometrial cancer cells, NUPR1, together with Nidogen 1 was found to be a target gene of the ETV5 transcription factor which is key to myoendometrial invasion by cancer cells (57). Eliminating NUPR1 in endometrial cells minimises cellular migration induced by ETV5 overexpression. NUPR1 was also shown to be highly expressed in the deep (invasive) endometrial cancers of the patients (57).

Myeloma. Using available GEO databases, Di Martino et al (2015) identified that the action of NUPR1 was one of the key mechanisms in myeloma progression and was connected with the downregulation of 6 genes, including BNIP3, GINS1, 
GRAMD3, KIF11, SHCBP1 and SPIN4 and the upregulation of the 2 genes, ELMODI and SLC16A6 (58). Bone marrow from multiple myeloma patients tends to have higher levels of NUPR1 transcript than healthy volunteers, although in that study the sample number was small (59). The silencing NUPR1 in myeloma cells also resulted in a reduction of cell proliferation and induction of apoptosis and cell cycle blockage (59).

Biliary cancers. In cholangiocarcinoma, NUPR1 is predominantly stained in the nucleus and more so in cancer cells (60). A human HuCCT1 cholangiocarcinoma cell was found to have a reduced rate of cell growth, and migration and invasiveness in response to EGF and serum, following NUPR1 knockdown by siRNA (60).

Hepatocellular carcinoma (HCC). TGF $\beta 1$ was able to markedly increase the expression of NUPR 1 in the Hep1 hepatocellular carcinoma cell line (8). In a pathway search study, NUPR1 was found amongst the top downregulated genes in non-viral HCCs, namely in alcohol consumption-related HCC (z ratio-3.0) and non-alcoholic fatty liver disease related HCC (z ratio-4.5) (61). HCC tissues tend to have high levels of NUPR1 protein, mostly in the nucleus, although no association was observed with TNM staging (12). Lee et al (2015) reported that NUPR1 was a key transcription regulator which leads to defective mitochondria-regulating genes in HCC (62). In this process, which is linked to metabolism of the cancer type, granulin is the key downstream effector protein of NUPR1. Knockdown of NUPR1 leads to a calcium signalling-dependent reduction of cellular invasion (62). Of note, the evaluation of NUPR1 mRNA expression in the limited clinical cohort only revealed a small portion of increase in NUPR1 transcript. NUPR 1 can be activated by hepatitis X protein (HBx) via the HBx-Smad4 pathway, which results in the reduction of cell death and the induction of vasculogenic mimicry in HCC (63). The same study demonstrated that NUPR1 transcript was found to be significantly upregulated in HCC compared with normal liver tissues by a ratio of 1.667. The knockdown of NUPR1 resulted in HCCs that were less mobile, had lower invasiveness and were less tumorigenic in vivo. It further identified the NUPR1/RELB/IER3/RUNX2 pathway as key in these events (12). In a comprehensive search for the transcriptomic and histone modification profiles during the transition from non-alcoholic steatohepatitis to HCC, it was found that NUPR1 plays an important role in this complex network (64). NUPR1 can inhibit lysine acetyltransferase 8 , which in turn influences one of the key generic alterations of gene patterns for the deacetylation of histone H4 Lysin 16 during the transition process. Serum from patients with chronic hepatitis $\mathrm{B}$ has been shown to be able to activate expression of NUPR1 in HCC cells (65). Thyroxin (T3) is a potent inducer of NUPR1 expression in HCC cell lines, by over 30 -fold, an effect attributable to the transcriptional regulation of thyroxin receptor protein directly binding and activating the transcriptional response elements of the NUPR1 promoter (66). Clinically, NUPR1 is positively associated with thyroxin receptors and the high levels of expression of both are significantly linked to shorter overall and disease-free survival (60).

Osteosarcoma. Osteosarcoma tissue has significantly higher levels of NUPR1 transcript compared with normal tissues (67). Together with miR4443, NUPR1 plays a regulatory role in a long non-coding RNA, FEZF1-AS1, induced cell growth, and the migration and invasiveness of osteosarcoma cells. IncRNA FAL1 and FEZF1-AS1 have been shown to require NUPR1 in their cancer inducing activities, acting respectively with miR637 and miR4443 $(39,67)$.

Neurological tumours. In gliomas, the NUPR1 transcript level is significantly higher than in normal tissues (68). High-grade glioma stains more strongly than normal brain tissue and low-grade tumours: Again, high levels of staining are associated with a shorter survival and also indicates NUPR1 to be an independent prognostic indicator (68). The study further confirmed that the knockdown of NUPR1 in multiple glioblastoma cells resulted in a reduction of cell migration and proliferation, and cell cycle arrest at the G0/G1 phase (69), events appearing to be coordinated by intracellular signalling events involving ERK1/2, p38 MAPK and cleavage of caspase-3 and p27 (68).

Pituitary tumours. There are currently no studies available on human tumours as yet, at least to the best of our knowledge. NUPR1 expression is generally quiescent in the pituitary gland. However, an animal study conducted by Mohammad et al (2004) demonstrated that a parent cell of $\mathrm{GH} 3$ somatolactotrope genotype and a gonadotropic pituitary cell, LbT2 was tumorigenic in nude mice, whereas when NUPR1 expression was reduced in these cell lines, it lost its ability to form tumours in vivo (70). It was subsequently established that at least in LbT2 gonadotropic pituitary cells, NUPR1 allows the cell to avoid the G0/G1 phase of the cell cycle (14) and that NUPR1 is transcriptively regulated by activating transcription factor 4 (ATF4), as in other cell types, such as HeLa cells $(71,72)$. The GCN/ATF4 pathway appears to involve the amino acid response element (AARE) with the NUPR1 promoter (73).

\section{NUPR1 in other conditions}

Neurological disorders. NUPR1 is one of the responsive genes in the cerebral cortex, which is downregulated in response to oestradiol (E2) induction (74). NUPR1, together with a few other proteins appears to be a key responsive molecule in neural cells and tissues following a challenge with methamphetamine (75). Treatment with this substance results in an increase in NUPR1 expression, which is associated with an increase in apoptosis and autophagy in neural cells in vivo and in cell lines in vitro (68).

Cardiac hypertrophy. Cardiac hypertrophy is the abnormal enlargement, or thickening, of the heart muscle, resulting from increases in cardiomyocyte size. It has been reported that NUPR1 is an important factor in cardiomyocyte hypertrophy, induced by endothelin and phenylephrine (76). NUPR1 is also key to transforming growth factor $\alpha$ (TNF $\alpha$ )-induced 
metalloproteinases in heart fibroblasts. These are key contributing factors in heart failure. It has also been observed that NUPR1 also partners with some of the muscle specific genes such as p68 (Ddx5) and MyoD in myoblasts (77).

Liver toxicity. NUPR1 is part of a protection mechanism in CCL4 [Chemokine (C-C motif) ligands 4] induced liver injury, by coordinating with cytochrome P450 2E1 which converts the chemical to toxic products (78).

Inflammation. The loss of NUPR1 in mice has been shown to result in increased death when challenged by lipopolysaccharide (LPS), together with an increase in TNF $\alpha$, and in the reactive oxygen species, myeloperoxidase and hydroperoxide, suggesting that the loss of protection of stress injuries by NUPR1 (27,79). Conversely, TNF $\alpha$ via NFkB mediates the expression of NUPR1 (80). It has also been indicated to be involved in the pathophysiological process of arthritis, in that osteoarthritic cartilage has higher levels of NUPR1 and that NUPR1 appears to be a key mediator in interleukin-1 $\beta$ induced MMP13 expression in chondrocytes (81).

Pancreatitis. It has been shown that LPS is able to rapidly induce the expression of NUPR la mRNA, detected by northern blot analysis in pancreatic acinar cells, in the pancreas, liver and kidneys (82). On the other hand, NUPR1 has been shown to coordinate with pancreatic associated protein-1 (PAP1) in protecting the pancreas from inflammation inducers (24). During chronic pancreatitis, NUPR1 is induced to express and protect pancreatic acinar cells from becoming apoptotic (83).

Diabetes. The loss of NUPR1 has also been observed with increases in the beta cell mass of the pancreas, suggesting that it is involved in glucose metabolism in the body (84). It has also been shown that NUPR1 plays a vital role in the protection of $\beta$-cells from apoptosis, related degradation of insulin storages and subsequent secretion during inflammatory and obesity-related tissue stress (82).

\section{Therapeutic considerations}

Therapeutic regulation of NUPR1. Bratland et al reported that vitamin $1,25(\mathrm{OH})_{2} \mathrm{D}_{3}$ was able to upregulate NUPR1 expression in MCF-7 breast cancer cells and in doing so, reduce colony formation and provoke cell cycle arrest at the G1 phase, attributable to the regulation of p21Kip1 (85).

Targeting. Trifluoperazine, a drug used for psychiatric conditions has been found to interact with NUPR1 and block NUPR1-dependent tumour growth. Novel derivatives from trifluoperazine, namely ZZW-115, have been synthesised since with improved binding and potent effects on pancreatic cancer cells, providing positive prospects of novel agents in therapeutically targeting NUPR1 $(7,86)$. Novel compounds that can target NUPR1 have been recently identified and shown to suppress pancreatic tumour development in the context of targeting NUPR1 (87). Although it is still too early to tell, it does indicate that NUPR1 would be a valuable therapeutic target in tumours closely linked to NUPR1 expression, pancreatic cancer being an excellent example. A recent study by Deng et al (2017) found that a STAT3 inhibitor, fluorofenidone was able to reduce the level of NUPR1 in lung adenocarcinoma cell lines, reproducibly demonstrated in vivo and in vitro, in line with reductions in cell growth, colony formation and tumour growth (88). Specific peptides have been shown to block the interaction between NUPR1 and one of its key partner proteins RING1B, indicating a value for targeting the action of NUPR1 (89). Cationic solid lipid nanoparticles have been tested as a means with which to deliver anti-NUPR1 plasmids to HCC cancer cells and have successfully resulted in reduction of NUPR1 in these cells (90). Moreover, Lan et al (2020) demonstrated that ZZW-115 sensitized cancer cells to genotoxic agents by the inhibition of NUPR1 nuclear translocation, which in turn reduced the SUMOylation-dependent functions of DNA damage response proteins (6).

Chemoresistance. NUPR1 has been found to be involved in the resistance of thyroid cancer cells to Lenvatinib therapy (91). Low levels of NUPR1 in fibroblasts have been shown to result in resistance to adriamycin (79). In breast cancer cells, NUPR1 also confers resistance to chemotherapeutic agents, including Taxol and doxorubicin, by involving the NUPR1-PI3K/Akt-phospho-p21 axis $(29,92)$. NUPR1-deficient pancreatic cancer cells are more sensitive to chemotherapeutic drugs, with the activation of NUPR1 increasing drug resistance to gemcitabine $(46,93)$ and also becoming more sensitive to HSP90 inhibitors (94) and mTOR inhibitors in squamous cell carcinoma of skin (95). Likewise, knockdown of NUPR1 in liver cancer cells sensitises their response to sorafenib, which itself induces the expression of NUPR1, resulting in acquired resistance $(12,66)$. However, the link may be more complex than just an NUPR1 connection. In a very interesting preliminary study, a small number of patients with cervical cancers were tested for changes in a panel of molecules involved in DNA repair and candidate drug resistance before and after two cycles of cisplatin treatment (96). NUPR1 was found to be one of the few proteins that was consistently reduced following treatment; however, this change was not connected to the clinical and pathological response. This important observation, the very first in a clinical setting, has raised a number of important questions; for example, the candidacy of NUPR 1 as a drug resistance regulator in the body in vitro for cisplatin, leads to the question of whether NUPR1 should be considered together with other key partners. The question is whether an increase in the reduction of NUPR1 is in fact a signal for drug resistance in the context of whole body for instance. This interesting research topic, to be expanded further by the researchers, would shed important light on such questions. In a colon cancer cell line (HCT116) spheroid model, NUPR1 was found to be markedly reduced (activation $\mathrm{z}$ ratio-1.387) in a full nutrient environment but less reduced ( $\mathrm{z}$ ratio-0.632) in a glucose deprived environment when cells were treated with irinotecan and chloroquine (97). Whilst this suggests that glucose is important in resistance to chemotherapy, it again raises questions as to the role of NUPR1 in the response to chemotherapy in different cancer types. In prostate cancer cell lines (DU145 and PC3) with acquired 
resistance to docetaxel, single-cell RNA-sequencing determined differential clusters of sensitive vs. resistant cells (98). Protein ubiquitination was the most differentially regulated pathway and one of the top regulators was identified to be NUPR1. NUPR1 gene modifications revealed that NUPR 1 conferred docetaxel resistance in both cell lines, indicating that it is a mediator of prostate cancer drug resistance and hence a target for resistance-reversal (98).

\section{Challenges and future perspectives}

NUPR1 is a highly interesting molecule to explore in the context of cancer, as demonstrated in the evidence gathered over the past 2 decades. It is involved in multiple aspects of cancer, from DNA repair, transcription regulation, cell cycle and death to metabolic activity of cancer cells. It also appears to be involved in a wide variety of cancer types. Clinically, there are demonstrable links between NUPR1 and disease progression and clinical outcome of patients, at least in certain cancer types. There are also early signs that it has a therapeutic value by targeting NUPR1 in the treatment of cancer. However, whilst exciting, a cautious approach is necessary until some of the key issues are resolved. The central issue is the inconsistencies in the role of NUPR1 in different cancer types and is a repeatedly occurring pattern. The following provides some perspectives for the likely reasons that this is observed.

Discrepancies in clinical and in vitro findings. Whilst NUPR1 itself has some contrasting roles in different types of cancer cells, a recently discovered family member, namely NUPR1-like or NUPR2, may provide some insight. NUPR1L closely resembles NUPR1 but has contrasting functions to NUPR1 $(5,99)$. NUPR2 is regulated by p53 responsive elements and its expression is dependent on p53, a classic tumour suppressor (5). NUPR2 expression is also induced by 53 inducers, including serum starvation and oxaliplatin. Furthermore, NUPR1 and NUPR2 mutually suppress each other by way of the transcription of regulation. NUPR 2 expression downregulates that of NUPR1 and vice versa. NUPR1 has been shown to be able to revert NUPR2 induced cell cycle arrest, making it a classic agonist-antagonist player in cells (5). NUPR2 is able to bind the same partners of NUPR1, including RING1B, at a similar affinity and most interestingly binds to NUPR1 (87). NUPR2 also appears to play an inhibitory role in colorectal cancer cells and can be targeted by miRF2277, which binds to the UTR of the human NUPR2 gene and downregulate NUPR2, and in doing so, increase the growth and migration of cancer cells (41). Thus, the clinical association between NUPR1 and cancer would require the consideration of NUPR2, in the same setting, namely in the same cancer types and the same cohort, in order to establish a solid link.

Nuclear vs. cytoplasmic. One of the most interesting observations from published studies is the cellular location of the protein. In normal follicles of the thyroid gland, NUPR1 exclusively displays nuclear staining. The same nuclear staining is seen in follicular tumours. However, papillary tumours largely exhibit cytoplasmic staining, particularly in large tumours and tumours with lymph node metastasis (47). In breast cancer, both cytoplasmic and nuclear staining was observed (31). These preliminary observations indicate that cytoplasmic distribution of NUPR1 in cancer cells may aid the aggressiveness and poor differentiation phenomenon of cancer cells. Pancreatic tumours also exhibit nuclear and cytoplasmic staining $(42,43)$. Again, heavy cytoplasmic staining was seen in NSCLC cancers (17). A study with a larger cohort enabling us to comprehensively evaluate the cellular location of NUPR1 would answer these questions. In addition, further cell-based investigations to discern the functional discrepancies for cytoplasmic NUPR1 and nuclear NUPR1 was thought to be necessary. The nuclear vs. cytoplasmic localisation of NUPR1 remained an enigma until a recent study by Lan et al (2020), whose elegant study on the effect of ZZW-115-dependent inhibition of NUPR 1 nuclear translocation by changes in SUMOylation-dependent functions of DNA damage response proteins (6).

A further point is that one should analyse NUPR1 together with its positive and negative regulators in the same study. For example, it would be invaluable to co-investigate NUPR1 and its potential partners such as ERBB2 in breast cancer (33). Finally, when considering targeting NUPR1 as a therapy, it is important to focus on the tumour type(s) that has/have a demonstrable connection, such as pancreatic cancer, whereas other cancer types would require additional work for the relationship to be firmly established. This is an exciting avenue of research that we anticipate could reveal new targets in cancer treatment in the future.

\section{Acknowledgements}

The authors would like to thank to the assistance of Dr Gregory Harrison (School of Medicine, Cardiff University) in formatting the manuscript.

\section{Funding}

No funding was received.

\section{Availability of data and materials}

Not applicable.

\section{Authors' contributions}

WGJ and TAM conceived the design of the study. AXL, AJS, $\mathrm{RH}, \mathrm{LY}$ and $\mathrm{KF}$ all contributed to the content (literature search and writing of the text) of the present review. All authors read and approved of the final manuscript.

\section{Ethics approval and consent to participate}

Not applicable.

\section{Patient consent for publication}

Not applicable.

\section{Competing interests}

The authors declare that they have no competing interests. 


\section{References}

1. Mallo GV, Fiedler F, Calvo EL, Ortiz EM, Vasseur S, Keim V, Morisset $\mathrm{J}$ and Iovanna JL: Cloning and expression of the rat $\mathrm{p} 8$ cDNA, a new gene activated in pancreas during the acute phase of pancreatitis, pancreatic development, and regeneration, and which promotes cellular growth. J Biol Chem 272: 32360-32369, 1997.

2. Vasseur S, Vidal Mallo G, Fiedler F, Bödeker H, Cánepa E, Moreno S and Iovanna JL: Cloning and expression of the human p8, a nuclear protein with mitogenic activity. Eur J Biochem 259: 670-675, 1999

3. Ree AH, Tvermyr M, Engebraaten O, Rooman M, Røsok O, Hovig E, Meza-Zepeda LA, Bruland OS and Fodstad O: Expression of a novel factor in human breast cancer cells with metastatic potential. Cancer Res 59: 4675-4680, 1999.

4. Quirk CC, Seachrist DD and Nilson JH: Embryonic expression of the luteinizing hormone $\beta$ gene appears to be coupled to the transient appearance of $\mathrm{p} 8$, a high mobility group-related transcription factor. J Biol Chem 278: 1680-1685, 2003.

5. Lopez MB, Garcia MN, Grasso D, Bintz J, Molejon MI, Velez G, Lomberk G, Neira JL, Urrutia R and Iovanna J: Functional characterization of Nupr1L, A Novel p53-regulated isoform of the high-mobility group (HMG)-related protumoral protein Nupr1. J Cell Physiol 230: 2936-2950, 2015.

6. Lan W, Santofimia-Castaño P, Swayden M, Xia Y, Zhou Z, Audebert S, Camoin L, Huang C, Peng L, Jiménez-Alesanco A, et al: ZZW-115-dependent inhibition of NUPR1 nuclear translocation sensitizes cancer cells to genotoxic agents. JCI Insight 5: e138117, 2020.

7. Santofimia-Castaño P, Rizzuti B, Xia Y, Abian O, Peng L, Velázquez-Campoy A, Iovanna JL and Neira JL: Designing and repurposing drugs to target intrinsically disordered proteins for cancer treatment: Using NUPR1 as a paradigm. Mol Cell Oncol 6: e1612678,2019.

8. Pommier RM, Gout J, Vincent DF, Cano CE, Kaniewski B, Martel S, Rodriguez J, Fourel G, Valcourt U, Marie JC, et al The human NUPR1/P8 gene is transcriptionally activated by transforming growth factor $\beta$ via the SMAD signalling pathway. Biochem J 445: 285-293, 2012

9. Hamidi T, Cano CE, Grasso D, Garcia MN, Sandi MJ, Calvo EL, Dagorn JC, Lomberk G, Urrutia R, Goruppi S, et al: Nupr1-aurora kinase A pathway provides protection against metabolic stress-mediated autophagic-associated cell death. Clin Cancer Res 18: 5234-5246, 2012.

10. Gironella M, Malicet C, Cano C, Sandi MJ, Hamidi T, Tauil RM, Baston M, Valaco P, Moreno S, Lopez F, et al: p8/nupr1 regulates DNA-repair activity after double-strand gamma irradiation-induced DNA damage. J Cell Physiol 221: 594-602, 2009.

11. Carracedo A, Lorente M, Egia A, Blázquez C, García S, Giroux V, Malicet C, Villuendas R, Gironella M, González-Feria L, et al: The stress-regulated protein $\mathrm{p} 8$ mediates cannabinoid-induced apoptosis of tumor cells. Cancer Cell 9: 301-312, 2006.

12. Emma MR, Iovanna JL, Bachvarov D, Puleio R, Loria GR, Augello G, Candido S, Libra M, Gulino A, Cancila V, et al: NUPR1, a new target in liver cancer: Implication in controlling cell growth, migration, invasion and sorafenib resistance. Cell Death Dis 7: e2269, 2016

13. Santofimia-Castaño P, Lan W, Bintz J, Gayet O, Carrier A Lomberk G, Neira JL, González A, Urrutia R, Soubeyran P and Iovanna J: Inactivation of NUPR1 promotes cell death by coupling ER-stress responses with necrosis. Sci Rep 8: 16999, 2018

14. Brannon KM, Million Passe CM, White CR, Bade NA, King MW and Quirk CC: Expression of the high mobility group A family member $\mathrm{p} 8$ is essential to maintaining tumorigenic potential by promoting cell cycle dysregulation in LbetaT 2 cells. Cancer Lett 254: 146-155, 2007.

15. Kong DK, Georgescu SP, Cano C, Aronovitz MJ, Iovanna JL, Patten RD, Kyriakis JM and Goruppi S: Deficiency of the transcriptional regulator $\mathrm{p} 8$ results in increased autophagy and apoptosis, and causes impaired heart function. Mol Biol Cell 21: $1335-1349,2010$

16. Li A, Li X, Chen X, Zeng C, Wang Z, Li Z and Chen J: NUPR1 Silencing induces autophagy-mediated apoptosis in multiple myeloma cells through the PI3K/AKT/mTOR pathway. DNA Cell Biol 39: 368-378, 2020.

17. Mu Y, Yan X, Li D, Zhao D, Wang L, Wang X, Gao D, Yang J, Zhang H, Li Y, et al: NUPR1 maintains autolysosomal efflux by activating SNAP25 transcription in cancer cells. Autophagy 14: 654-670, 2018
18. Grasso D, Garcia MN, Hamidi T, Cano C, Calvo E, Lomberk G, Urrutia $R$ and Iovanna JL: Genetic inactivation of the pancreatitis-inducible gene Nuprl impairs PanIN formation by modulating KrasG12D-induced senescence. Cell Death Differ 21: 1633-1641, 2014.

19. Grasso D, Bintz J, Lomberk G, Molejon MI, Loncle C, Garcia MN, Lopez MB, Urrutia R and Iovanna JL: Pivotal role of the chromatin protein Nupr1 in Kras-induced senescence and transformation. Sci Rep 5: 17549, 2015.

20. Cai D, Huang E, Luo B, Yang Y, Zhang F, Liu C, Lin Z, Xie WB and Wang H: Nuprl/Chop signal axis is involved in mitochondrion-related endothelial cell apoptosis induced by methamphetamine. Cell Death Dis 7: e2161, 2016.

21. Tang B, Li Q, Zhao XH, Wang HG, Li N, Fang Y, Wang K, Jia YP, Zhu P, Gu J, et al: Shiga toxins induce autophagic cell death in intestinal epithelial cells via the endoplasmic reticulum stress pathway. Autophagy 11: 344-354, 2015

22. Shiraki M, Xu X, Iovanna JL, Kukita T, Hirata $H$, Kamohara A, Kubota Y, Miyamoto H, Mawatari M and Kukita A: Deficiency of stress-associated gene Nuprl increases bone volume by attenuating differentiation of osteoclasts and enhancing differentiation of osteoblasts. FASEB J 33: 8836-8852, 2019.

23. Maida A, Zota A, Sjøberg KA, Schumacher J, Sijmonsma TP, Pfenninger A, Christensen MM, Gantert T, Fuhrmeister J, Rothermel U, et al: A liver stress-endocrine nexus promotes metabolic integrity during dietary protein dilution. J Clin Invest 126: 3263-3278, 2016.

24. Hoffmeister A, Ropolo A, Vasseur S, Mallo GV, Bodeker H, Ritz-Laser B, Dressler GR, Vaccaro MI, Dagorn JC, Moreno S and Iovanna JL: The HMG-I/Y-related protein p8 binds to p300 and Pax 2 trans-activation domain-interacting protein to regulate the trans-activation activity of the Pax $2 \mathrm{~A}$ and Pax2B transcription factors on the glucagon gene promoter. J Biol Chem 277: 22314-22319, 2002.

25. Päth G, Opel A, Knoll A and Seufert J: Nuclear protein p8 is associated with glucose-induced pancreatic beta-cell growth. Diabetes 53 (Suppl 1): S82-S85, 2004.

26. Zhong C, Yu J, Li D, Jiang K, Tang Y, Yang M, Shen H, Fang X, Ding K, Zheng S and Yuan Y: Zyxin as a potential cancer prognostic marker promotes the proliferation and metastasis of colorectal cancer cells. J Cell Physiol: Jan 29, 2019 (Epub ahead of print).

27. Vasseur S, Hoffmeister A, Garcia S, Bagnis C, Dagorn JC and Iovanna JL: $\mathrm{p} 8$ is critical for tumour development induced by rasV12 mutated protein and E1A oncogene. EMBO Rep 3: $165-170,2002$

28. Iovanna JL: Expression of the stress-associated protein $\mathrm{p} 8$ is a requisite for tumor development. Int J Gastrointest Cancer 31: 89-98, 2002.

29. Clark DW, Mitra A, Fillmore RA, Jiang WG, Samant RS, Fodstad O and Shevde LA: NUPR1 interacts with p53, transcriptionally regulates p21 and rescues breast epithelial cells from doxorubicin-induced genotoxic stress. Curr Cancer Drug Targets 8: 421-430,2008.

30. Simpson NE, Lambert WM, Watkins R, Giashuddin S, Huang SJ, Oxelmark E, Arju R, Hochman T, Goldberg JD, Schneider RJ, et al: High levels of Hsp90 cochaperone p23 promote tumor progression and poor prognosis in breast cancer by increasing lymph node metastases and drug resistance. Cancer Res 70: 8446-8456, 2010.

31. Jiang WG, Watkins G, Douglas-Jones A, Mokbel K, Mansel RE and Fodstad O: Expression of Com-1/P8 in human breast cancer and its relevance to clinical outcome and ER status. Int J Cancer 117: 730-737, 2005.

32. Jiang WG, Davies G and Fodstad O: Com-1/P8 in oestrogen regulated growth of breast cancer cells, the ER-beta connection. Biochem Biophys Res Commun 330: 253-262, 2005.

33. Jung SH, Lee A, Yim SH, Hu HJ, Choe C and Chung YJ: Simultaneous copy number gains of NUPR1 and ERBB2 predicting poor prognosis in early-stage breast cancer. BMC Cancer 12: 382, 2012.

34. Hollern DP, Honeysett J, Cardiff RD and Andrechek ER: The E2F transcription factors regulate tumor development and metastasis in a mouse model of metastatic breast cancer. Mol Cell Biol 34: 3229-3243, 2014.

35. Jia Q, Zhou W, Yao W, Yang F, Zhang S, Singh R, Chen J, Chen JJ, Zhang Y, Wei F, et al: Downregulation of YAP-dependent Nuprl promotes tumor-repopulating cell growth in soft matrices. Oncogenesis 5: e220, 2016. 
36. Fish L, Zhang S, Yu JX, Culbertson B, Zhou AY, Goga A and Goodarzi H: Cancer cells exploit an orphan RNA to drive metastatic progression. Nat Med 24: 1743-1751, 2018.

37. Matsunaga K, Fujisawa K, Takami T, Burganova G, Sasai N, Matsumoto T, Yamamoto N and Sakaida I: NUPR1 acts as a pro-survival factor in human bone marrow-derived mesenchymal stem cells and is induced by the hypoxia mimetic reagent deferoxamine. J Clin Biochem Nutr 64 : 209-216, 2019

38. Davies ML, Parr C, Sanders AJ, Fodstad O and Jiang WG: The transcript expression and protein distribution pattern in human colorectal carcinoma reveal a pivotal role of COM-1/p8 as a tumour suppressor. Cancer Genomics Proteomics 7: 75-80, 2010.

39. Wang L, Jiang F, Xia $X$ and Zhang B: LncRNA FAL1 promotes carcinogenesis by regulation of miR-637/NUPR1 pathway in colorectal cancer. Int J Biochem Cell Biol 106: 46-56, 2019.

40. Li X, Martin TA and Jiang WG: COM-1/p8 acts as a tumour growth enhancer in colorectal cancer cell lines. Anticancer Res 32: 1229-1237, 2012.

41. Gao Q, Lei F, Zeng Q, Gao Z, Niu P, Junnan, Ning, Li J and Zhang J: Functional passenger-strand miRNAs in exosomes derived from human colon cancer cells and their heterogeneous paracrine effects. Int J Biol Sci 16: 1044-1058, 2020

42. Su SB, Motoo Y, Iovanna JL, Berthézène P, Xie MJ, Mouri H, Ohtsubo K, Matsubara F and Sawabu N: Overexpression of p8 is inversely correlated with apoptosis in pancreatic cancer. Clin Cancer Res 7: 1320-1324, 2001.

43. Su SB, Motoo Y, Iovanna JL, Xie MJ, Mouri H, Ohtsubo K, Yamaguchi Y, Watanabe H, Okai T, Matsubara F and Sawabu N: Expression of p8 in human pancreatic cancer. Clin Cancer Res 7: 309-313, 2001.

44. Hamidi T, Algül H, Cano CE, Sandi MJ, Molejon MI, Riemann M, Calvo EL, Lomberk G, Dagorn JC, Weih F, et al: Nuclear protein 1 promotes pancreatic cancer development and protects cells from stress by inhibiting apoptosis. J Clin Invest 122: 2092-2103, 2012.

45. Cano CE, Hamidi T, Sandi MJ and Iovanna JL: Nupr1: The Swiss-knife of cancer. J Cell Physiol 226: 1439-1443, 2011.

46. Cano CE, Hamidi T, Garcia MN, Grasso D, Loncle C, Garcia S, Calvo E, Lomberk G, Dusetti N, Bartholin L, et al: Genetic inactivation of Nuprl acts as a dominant suppressor event in a two-hit model of pancreatic carcinogenesis. Gut 63: 984-995, 2014

47. Ito Y, Yoshida H, Motoo Y, Miyoshi E, Iovanna JL, Tomoda C, Uruno T, Takamura Y, Miya A, Kobayashi K, et al: Expression and cellular localization of $\mathrm{p} 8$ protein in thyroid neoplasms. Cancer Lett 201: 237-244, 2003.

48. Ito Y, Yoshida H, Motoo Y, Iovanna JL, Tomoda C, Uruno T, Takamura Y, Miya A, Kobayashi K, Matsuzuka F, et al: Expression of p8 protein in medullary thyroid carcinoma. Anticancer Res 25: 3419-3423, 2005.

49. Jiang WG, Davies G, Martin TA, Kynaston H, Mason MD and Fodstad O: Com-1/p8 acts as a putative tumour suppressor in prostate cancer. Int J Mol Med 18: 981-986, 2006.

50. Du P, Ye L, Yang Y and Jiang WG: Candidate of metastasis 1 regulates in vitro growth and invasion of bladder cancer cells. Int J Oncol 42: 1249-1256, 2013.

51. Veerla S, Panagopoulos I, Jin Y, Lindgren D and Höglund M: Promoter analysis of epigenetically controlled genes in bladder cancer. Genes Chromosomes Cancer 47: 368-378, 2008

52. Guo X, Wang W, Hu J, Feng K, Pan Y, Zhang L and Feng Y: Lentivirus-mediated RNAi knockdown of NUPR1 inhibits human nonsmall cell lung cancer growth in vitro and in vivo. Anat Rec (Hoboken) 295: 2114-2121, 2012

53. Ghandhi SA, Ponnaiya B, Panigrahi SK, Hopkins KM, Cui Q, Hei TK, Amundson SA and Lieberman HB: RAD9 deficiency enhances radiation induced bystander DNA damage and transcriptomal response. Radiat Oncol Lond Engl 9: 206, 2014

54. Wu H, Wang W and Xu H: Depletion of C3orf1/TIMMDC1 inhibits migration and proliferation in 95D lung carcinoma cells. Int J Mol Sci 15: 20555-20571, 2014

55. Frances D, Sharma N, Pofahl R, Maneck M, Behrendt K, Reuter K, Krieg T, Klein CA, Haase I and Niemann C: A role for Rac1 activity in malignant progression of sebaceous skin tumors. Oncogene 34: 5505-5512, 2015.

56. Niessner H, Sinnberg T, Kosnopfel C, Smalley KSM, Beck D, Praetorius C, Mai M, Beissert S, Kulms D, Schaller M, et al: BRAF inhibitors amplify the proapoptotic activity of MEK inhibitors by inducing ER stress in NRAS-mutant melanoma. Clin Cancer Res 23: 6203-6214, 2017.
57. Pedrola N, Devis L, Llauradó M, Campoy I, Martinez-Garcia E, Garcia M, Muinelo-Romay L, Alonso-Alconada L, Abal M, Alameda F, et al: Nidogen 1 and Nuclear Protein 1: Novel targets of ETV5 transcription factor involved in endometrial cancer invasion. Clin Exp Metastasis 32: 467-478, 2015.

58. Di Martino MT, Guzzi PH, Caracciolo D, Agnelli L, Neri A, Walker BA, Morgan GJ, Cannataro M, Tassone P and Tagliaferri P: Integrated analysis of microRNAs, transcription factors and target genes expression discloses a specific molecular architecture of hyperdiploid multiple myeloma. Oncotarget 6: 19132-19147, 2015

59. Zeng C, Li X, Li A, Yi B, Peng X, Huang X and Chen J: Knockdown of NUPR1 inhibits the growth of U266 and RPMI8226 multiple myeloma cell lines via activating PTEN and caspase activation-dependent apoptosis. Oncol Rep 40: $1487-1494,2018$

60. Kim KS, Jin DI, Yoon S, Baek SY, Kim BS and Oh SO: Expression and roles of NUPR1 in cholangiocarcinoma cells. Anat Cell Biol 45: 17-25, 2012

61. Seshachalam VP, Sekar K and Hui KM: Insights into the etiology-associated gene regulatory networks in hepatocellular carcinoma from The Cancer Genome Atlas. J Gastroenterol Hepatol 33: 2037-2047, 2018

62. Lee YK, Jee BA, Kwon SM, Yoon YS, Xu WG, Wang HJ, Wang XW, Thorgeirsson SS, Lee JS, Woo HG and Yoon G: Identification of a mitochondrial defect gene signature reveals NUPR1 as a key regulator of liver cancer progression. Hepatology 62: 1174-1189, 2015.

63. Bak Y, Shin H, Bak I seon, Yoon D and Yu DY: Hepatitis B virus $\mathrm{X}$ promotes hepatocellular carcinoma development via nuclear protein 1 pathway. Biochem Biophys Res Commun 466: 676-681, 2015.

64. de Conti A, Dreval K, Tryndyak V, Orisakwe OE, Ross SA, Beland FA and Pogribny IP: Inhibition of the cell death pathway in nonalcoholic steatohepatitis (NASH)-related hepatocarcinogenesis is associated with histone $\mathrm{H} 4$ lysine 16 deacetylation. Mol Cancer Res 15: 1163-1172, 2017.

65. Ji Y, Wang Z, Chen H, Zhang L, Zhuo F and Yang Q: Serum from chronic hepatitis $\mathrm{B}$ patients promotes growth and proliferation via the IGF-II/IGF-IR/MEK/ERK signaling pathway in hepatocellular carcinoma cells. Cell Physiol Biochem 47: 39-53, 2018.

66. Chen CY, Wu SM, Lin YH, Chi HC, Lin SL, Yeh CT, Chuang WY and Lin KH: Induction of nuclear protein-1 by thyroid hormone enhances platelet-derived growth factor A mediated angiogenesis in liver cancer. Theranostics 9: 2361-2379, 2019

67. Zhou C, Xu J, Lin J, Lin R, Chen K, Kong J and Shui X: Long non-coding RNA FEZF1-AS1 promotes osteosarcoma progression by regulating miR-4443/NUPR1 axis. Oncol Res 26: 1335-1343, 2018.

68. Li J, Ren S, Liu Y, Lian Z, Dong B, Yao Y and Xu Y: Knockdown of NUPR1 inhibits the proliferation of glioblastoma cells via ERK1/2, p38 MAPK and caspase-3. J Neurooncol 132: 15-26, 2017.

69. Li J, Lian ZG, Xu YH, Liu RY, Wei ZQ, Li T, Lv HT, Zhao YS, Liu YJ, Dong B and Fu X: Downregulation of nuclear protein-1 induces cell cycle arrest in G0/G1 phase in glioma cells in vivo and in vitro via P27. Neoplasma 67: 843-850, 2020.

70. Mohammad HP, Seachrist DD, Quirk CC and Nilson JH Reexpression of $\mathrm{p} 8$ contributes to tumorigenic properties of pituitary cells and appears in a subset of prolactinomas in transgenic mice that hypersecrete luteinizing hormone. Mol Endocrinol 18: 2583-2593, 2004.

71. Passe CM, Cooper G and Quirk CC: The murine p8 gene promoter is activated by activating transcription factor 4 (ATF4) in the gonadotrope-derived LbetaT2 cell line. Endocrine 30 81-91, 2006.

72. Jin HO, Seo SK, Woo SH, Choe TB, Hong SI, Kim JI and Park IC: Nuclear protein 1 induced by ATF4 in response to various stressors acts as a positive regulator on the transcriptional activation of ATF4. IUBMB Life 61: 1153-1158, 2009.

73. Averous J, Lambert-Langlais S, Cherasse Y, Carraro V, Parry L, B'chir W, Jousse C, Maurin AC, Bruhat A and Fafournoux P: Amino acid deprivation regulates the stress-inducible gene $\mathrm{p} 8$ via the GCN2/ATF4 pathway. Biochem Biophys Res Commun 413: 24-29, 2011

74. Sárvári M, Kalló I, Hrabovszky E, Solymosi N, Tóth K, Likó I, Molnár B, Tihanyi K and Liposits Z: Estradiol replacement alters expression of genes related to neurotransmission and immune surveillance in the frontal cortex of middle-aged, ovariectomized rats. Endocrinology 151: 3847-3862, 2010. 
75. Xu X, Huang E, Tai Y, Zhao X, Chen X, Chen C, Chen R, Liu C, Lin Z, Wang $H$ and Xie WB: Nupr1 modulates methamphetamine-induced dopaminergic neuronal apoptosis and autophagy through CHOP-Trib3-mediated endoplasmic reticulum stress signaling pathway. Front Mol Neurosci 10: 203, 2017.

76. Goruppi S, Patten RD, Force T and Kyriakis JM: Helix-loop-helix protein $\mathrm{p} 8$, a transcriptional regulator required for cardiomyocyte hypertrophy and cardiac fibroblast matrix metalloprotease induction. Mol Cell Biol 27: 993-1006, 2007.

77. Sambasivan R, Cheedipudi S, Pasupuleti N, Saleh A, Pavlath GK and Dhawan J: The small chromatin-binding protein $\mathrm{p} 8$ coordinates the association of anti-proliferative and pro-myogenic proteins at the myogenin promoter. J Cell Sci 122: 3481-3491, 2009.

78. Taïeb D, Malicet C, Garcia S, Rocchi P, Arnaud C, Dagorn JC, Iovanna JL and Vasseur S: Inactivation of stress protein $\mathrm{p} 8$ increases murine carbon tetrachloride hepatotoxicity via preserved CYP2E1 activity. Hepatology 42: 176-182, 2005.

79. Vasseur S, Hoffmeister A, Garcia-Montero A, Mallo GV, Feil R, Kühbandner S, Dagorn JC and Iovanna JL: p8-deficient fibroblasts grow more rapidly and are more resistant to adriamycin-induced apoptosis. Oncogene 21: 1685-1694, 2002.

80. Kallwellis K, Grempler R, Günther S, Päth G and Walther R: Tumor necrosis factor alpha induces the expression of the nuclear protein $\mathrm{p} 8$ via a novel NF kappaB binding site within the promoter. Horm Metab Res 38: 570-574, 2006.

81. Yammani RR and Loeser RF: Brief report: Stress-inducible nuclear protein 1 regulates matrix metalloproteinase 13 expression in human articular chondrocytes. Arthritis Rheumatol 66 1266-1271, 2014.

82.Jiang YF, Vaccaro MI, Fiedler F, Calvo EL and Iovanna JL: Lipopolysaccharides induce p8 mRNA expression in vivo and in vitro. Biochem Biophys Res Commun 260: 686-690, 1999.

83. Motoo Y, Iovanna JL, Mallo GV, Su SB, Xie MJ and Sawabu N: $\mathrm{P} 8$ expression is induced in acinar cells during chronic pancreatitis. Dig Dis Sci 46: 1640-1646, 2001.

84. Hollenbach M, Klöting N, Sommerer I, Lorenz J, Heindl M, Kern M, Mössner J, Blüher M and Hoffmeister A: p8 deficiency leads to elevated pancreatic beta cell mass but does not contribute to insulin resistance in mice fed with high-fat diet. PLoS One 13: e0201159, 2018.

85. Bratland A, Risberg K, Maelandsmo GM, Gützkow KB, Olsen OE, Moghaddam A, Wang MY, Hansen CM, Blomhoff HK, Berg JP, et al: Expression of a novel factor, com1, is regulated by 1,25 -dihydroxyvitamin D 3 in breast cancer cells. Cancer Res 60: 5578-5583, 2000

86. Santofimia-Castaño P, Xia Y, Lan W, Zhou Z, Huang C, Peng L, Soubeyran $\mathrm{P}$, Velázquez-Campoy A, Abián O, Rizzuti B, et al: Ligand-based design identifies a potent NUPR1 inhibitor exerting anticancer activity via necroptosis. J Clin Invest 129 : 2500-2513, 2019.

87. Neira JL, Bintz J, Arruebo M, Rizzuti B, Bonacci T, Vega S, Lanas A, Velázquez-Campoy A, Iovanna JL and Abián O: Identification of a drug targeting an intrinsically disordered protein involved in pancreatic adenocarcinoma. Sci Rep 7: 39732, 2017.

88. Deng ZH, Meng J, Tang J, Hu GY and Tao LJ: Fluorofenidone Inhibits the Proliferation of Lung Adenocarcinoma Cells. J Cancer 8: 1917-1926, 2017.
89. Santofimia-Castaño P,Rizzuti B, Abián O,Velázquez-Campoy A Iovanna JL and Neira JL: Amphipathic helical peptides hamper protein-protein interactions of the intrinsically disordered chromatin nuclear protein 1 (NUPR1). Biochim Biophys Acta Gen Subj 1862: 1283-1295, 2018

90. Botto C, Augello G, Amore E, Emma MR, Azzolina A, Cavallaro G, Cervello M and Bondì ML: Cationic solid lipid nanoparticles as non viral vectors for the inhibition of hepatocellular carcinoma growth by RNA interference. J Biomed Nanotechnol 14: 1009-1016, 2018.

91. Khan HY, Ge J, Nagasaka M, Aboukameel A, Mpilla G, Muqbil I, Szlaczky M, Chaker M, Baloglu E, Landesman Y, et al: Targeting XPO1 and PAK4 in 8505C anaplastic thyroid cancer cells: Putative implications for overcoming lenvatinib therapy resistance. Int J Mol Sci 21: 237, 2019.

92. Vincent AJ, Ren S, Harris LG, Devine DJ, Samant RS, Fodstad O and Shevde LA: Cytoplasmic translocation of p21 mediates NUPR1-induced chemoresistance: NUPR1 and p21 in chemoresistance. FEBS Lett 586: 3429-3434, 2012.

93. Palam LR, Gore J, Craven KE, Wilson JL and Korc M: Integrated stress response is critical for gemcitabine resistance in pancreatic ductal adenocarcinoma. Cell Death Dis 6: e1913, 2015.

94. Augello G, Emma MR, Cusimano A, Azzolina A, Mongiovì S, Puleio R, Cassata G, Gulino A, Belmonte B, Gramignoli R, et al: Targeting HSP90 with the small molecule inhibitor AUY922 (luminespib) as a treatment strategy against hepatocellular carcinoma. Int J Cancer 144: 2613-2624, 2019.

95. Yu SL, Lee DC, Baek SW, Cho DY, Choi JG and Kang J: Identification of mTOR inhibitor-resistant genes in cutaneous squamous cell carcinoma. Cancer Manag Res 10: 6379-6389, 2018.

96. Real NE, Castro GN, Darío Cuello-Carrión F, Perinetti C, Röhrich H, Cayado-Gutiérrez N, Guerrero-Gimenez ME and Ciocca DR: Molecular markers of DNA damage and repair in cervical cancer patients treated with cisplatin neoadjuvant chemotherapy: An exploratory study. Cell Stress Chaperones 22: 811-822, 2017.

97. Schroll MM, LaBonia GJ, Ludwig KR and Hummon AB: Glucose restriction combined with autophagy inhibition and chemotherapy in HCT 116 spheroids decreases cell clonogenicity and viability regulated by tumor suppressor genes. J Proteome Res 16: 3009-3018, 2017.

98. Schnepp PM, Shelley G, Dai J, Wakim N, Jiang H, Mizokami A and Keller ET: Single-cell transcriptomics analysis identifies nuclear protein 1 as a regulator of docetaxel resistance in prostate cancer cells. Mol Cancer Res 18: 1290-1301, 2020

99. Urrutia R, Velez G, Lin M, Lomberk G, Neira JL and Iovanna J: Evidence supporting the existence of a NUPR1-like family of helix-loop-helix chromatin proteins related to, yet distinct from, AT hook-containing HMG proteins. J Mol Model 20: 2357, 2014.

100. Ree AH, Pacheco MM, Tvermyr M, Fodstad O and Brentani MM: Expression of a novel factor, com1, in early tumor progression of breast cancer. Clin Cancer Res 6: 1778-1783, 2000.

101. Cano CE, Sandí MJ, Hamidi T, Calvo EL, Turrini O, Bartholin L, Loncle C, Secq V, Garcia S, Lomberk G, et al: Homotypic cell cannibalism, a cell-death process regulated by the nuclear protein 1, opposes to metastasis in pancreatic cancer. EMBO Mol Med 4: 964-979, 2012. 